\title{
Microbial Spectrum of Intra-Abdominal Pathology of Patients Requiring Surgical Intervention in a Rural Teaching Hospital
}

\author{
Thomas K Joy ${ }^{1}$,Vergis Paul ${ }^{2}$,Jomine Jose ${ }^{3}$, Anna Mathew ${ }^{4}$, T.M Jacob ${ }^{5}$, \\ Celine $\mathrm{T} \mathrm{M}^{6}$
}

${ }^{1}$ III Year MBBS, ${ }^{2}$ Professor, ${ }^{3}$ Associate Professor, Department of General Surgery, ${ }^{4}$ Research Coordinator, MOSC Medical College, Kolenchery, ${ }^{5}$ Associate Professor, Department of Statistics, Nirmala College, Muvattupuzha, ${ }^{6}$ Associate Professor, Department of Community Medicine, MOSC Medical College, Kolenchery, Kerala, India.

\begin{abstract}
:
Background: Intra abdominal pathologies requiring surgical intervention include appendicitis, malignancies, trauma , perforation peritonitis etc. The most common organism isolated at the time of surgery was E. coli.We studied the spectrum of microbes at the time of laparotomy/laparoscopy to help us to know the emerging trends in microbiological spectrum and the sensitivity pattern in this medical college hospital.

Objective: To study the microbial spectrum and sensitivity pattern in patients with intra abdominal pathology requiring surgical interventions.

Methodology: After ethical committee approval was obtained, data for this retrospective study, with a sample size of 93 cases were collected from theGeneral Surgery Records and analysed.

Result: Of the 93 cases studied, E. coli dominated in 75\% cases of acute appendicitis and Enterococcus was found in $16.2 \%$. In cases of hollow viscous perforation 50\% were E.coli and $15.3 \%$ were K.pneumoniae. In acute appendicitis with peritonitis $64.25 \%$ were E.coli, K.pneumoniae and Enterococcus were $21.4 \%$ each.In case of colonic malignancy $28.5 \%$ of patients were infected by E. coli followed by enterococcus with $21.4 \%$. E.coli and K. pneumoniae were more sensitive to carbapenems, colistin and fluroquinolones, while Enterococcus was more sensitive to Linezolid.

Conclusion: E. coli is the organism that predominates in intra abdominal pathology requiringsurgical intervention and the common organisms are mostly sensitive to the carbapenems, linezolid, Colistin and fluroquinolones.
\end{abstract}

Keywords: Microbial spectrum, microbial sensitivity, E. coli, Meropenem,

\section{Introduction}

Intra-abdominal pathologies are many and varied, ranging from appendicitis, malignancies, trauma, perforation peritonitis etc. They are generally the result of invasion and multiplication of enteric bacteria in the wall of a hollow viscous or beyond. Escherichia coli is the most common organism isolated from the patients who have intra-abdominal pathology ${ }^{1}$.

Carbapenems retained the highest susceptibility rates against hospital and community acquired E.coli and K.pneumoniae pathologies ${ }^{2}$.

The microbiology of intra-abdominal pathologies is significantly altered in patients who have been exposed to the health care setting. This alteration may be due to the acquisition of nosocomial pathogens or may reflect prior antimicrobial therapy that has selected for resistant organisms. In a study by K. Krobot et al.(2004) ${ }^{3}$ it was found that appendicular pathology was the most common intra-abdominal pathology with $38 \%$, colonic pathologies accounted with 26\%, duodenal pathologies with $23 \%$ and biliary pathologies for which cholecystectomy was done accounted to about $6 \%$.

In a study by D.H Wittmann et al. (1991) ${ }^{4}$, it was found that out of 900 isolates from intra abdominal pathologies, E.coli was the most common aerobe isloated. It was isloated from about 450 patients with intra abdominal pathologies. K. pneumoniae was isloated from about 110 patients.Both Enterobacter spp. and Pseudomonas spp. accounted in less than 100 cases of intra abdominal pathology. In anaerobic organisms Bacteroides was isolated in about more than 600 cases.

In a study by M. Guembe et al. (2003-2007) ${ }^{5}$,a total of 572 aerobic and facultative gram-negative bacilli were isolated from intra-abdominal infections in 510 patients. E.coli $(52 \%)$ was the most common isolated species, followed by Klebsiella spp. (16\%), Proteus mirabilis (6.3\%) and Enterobacter spp. (6.3\%). Among the non-fermenters, P. aeruginosa was the most common isolated species (6.8\%).

There has been similar studiesin the past like D.H Wittmann et al.(1991) ${ }^{4}$, who showed that cefotaxime, cefoperazone and moxalactam were sensitive in $100 \%$ of cases for E.coli. this was followed by cefotetan with 
$99 \%$ and imipenem with $98 \%$. Ceftazidime was sensitive in $100 \%$ of cases which were followed by cefotaxime and cefotetan with $99 \%$ sensitivity for K.pneumoniae.

In a rural tertiary care centre there has not been much record of literature about the microbial spectrum and sensitivity pattern of intra-abdominal pathologies which led us to selecting this topic.

This project is aimed at studying the spectrum of microbes, at the time of laparotomy/laparoscopy and their sensitivity pattern. This would help us know the microbiological spectrum and the sensitivity pattern and would help us to know the emerging trends in sensitivity and resistance and also in anticipating the antimicrobial therapy.

\section{Methodology}

The study was a retrospective review carried out in the department of General Surgery of MOSC Medical College, Kolenchery during $1^{\text {st }}$ June 2014 to $31^{\text {st }}$ July 2014. Study Population: 93 patients above 12 years of age who underwent laparotomy/laparoscopy and had peritoneal swab or peritoneal pus which has been sent for culture sensitivity intra-operatively during the period April 2009 to April 2014 were studied. The patients were stratified into different pathological groups based on the microbe present and the anti-microbial sensitivity.

Data collection: All data, including microbial culture sensitivity report were collected from patient records and entered into case study sheet. The initial antibiotic used was recorded and the changes in the antimicrobial therapy based on the culture sensitivity pattern were noted. The various post-operative morbidities were also studied.

Ethical Issues: Waiver of informed consent was requested as patients were not going to be interviewed and observations were made only from the hospital records for which permission was obtained from the medical superintendent.The study was approved by the ethics committee on 15.01.2014. All records were maintained anonymously and kept strictly confidential by the investigator.

Intra-abdominal pathology

\section{Results}

\section{Acute appendicitis :}

A total of 44 cases of acute appendicitis were studied. E.coli was the most common organism isolated from acute appendicitis with $52.3 \%(\mathrm{p}=0.0008)$, followed by Enterococcus spp. and K. pneumoniae with $6.8 \%$. In combination Enterococcus spp. and E.coli with 9\%.

\section{Hollow viscous perforation peritonitis :}

A total of 16 cases of hollow viscous perforation peritonitis were studied. E.coli was the most common organism isolated from hollow viscous perforation peritonitis with $31.25 \%(\mathrm{p}=0.4142)$, followed by $K$. pneumonia with $18.75 \%$. In combination, K. pneumonia and E. coli, and Enterobacter spp. and E.coli with $6.2 \%$.

\section{Acute appendicitis with peritonitis :}

A total of 14 cases of acute appendicitis with peritonitis were studied. E.coli was the most common organism isolated from acute appendicitis with peritonitis with $42.8 \%$ ( $\mathrm{p}=0.0943$ ), followed by P.aeruginosa with 14.4\%. In combination, K.peumoniae and E.coli, Enterococcus spp. and E.coli, and Enterococcus spp. and $K$. pneumoniae with $7.1 \%$.

Colorectal surgery:

A total of 12 colorectal cases were studied. E.coli was the most common organism isolated from colorectal surgery with $25 \%(\mathrm{p}=0.4895)$, followed by Enterococcus species with $16.6 \%$. In combination Enterococcus spp.and K. peumoniae accounted to $16.6 \%$.

Cholilithiasis, choledocolithiasis:

A total of 4 cases of cholilithiasis, choledocolithiasis were studied. Enterobacter spp. was the most common organism isolated from cholilithiasis and choledocolithiasis with $50 \%(\mathrm{p}=0.4652)$, followed by E.coli with 25\%. In combination, K.pneumoniae and Streptococci spp. with $25 \%$.

Carcinoma stomach :

A total of 2 cases of carcinoma stomach were studied. Acinetobacter spp. was the most common organism isolated from carcinoma stomach with 50\%. In combination Enterobacter spp. and Streptococci with $50 \%$.

Intussusception : There was 1 case of intussusception. In intussusception E.coli was isolated with $100 \%$ presence.

Sensitivity pattern of organisms in intra abdominal pathology

\section{Acinetobacter spp. :}

Tigecycline was sensitive in $14.2 \%$ of cases of Acinetobacter spp infections. In combination, colistin and tigecycline were sensitive in $28.5 \%$, colistin and tobramycin, ciprlfloxacin and cephalexin, and colistin and 
meropenem in $14.2 \%$ of cases..

\section{Enterobacter spp. :}

In combination linezolid and vancomycin were sensitive in $50 \%$ of cases and gentamicin and ciprofloxacin in $33.3 \%$ of cases of Enterobacter spp. Infections.

\section{Enterococcus spp. :}

Vancomycin was sensitive in $6.2 \%$ of cases of Enterococcus spp. infections. In combination linezolid and vancomycin sensitive in 50\%, ampicillin and cephalexin in 12.5\%, and linezolid and erythromycin in $6.2 \%$. K.pneumoniae:

Colistin was sensitive in $11.8 \%$ to K.pneumoniae. In combination doripenem and imipenemwas sensitive in $17.6 \%$, and imipenem and meropenem in $11.8 \%$.

\section{E.coli :}

In combination doripenem and imipenem were sensitive in $19.3 \%$, colistin and meropenem in $17.5 \%$, imipenem and meropenem in $10.5 \%$, meropenem and tigecycline in $14 \%$, and ciprofloxacin and meropenem in $8.8 \%$ to E.coli.

Streptococci :

Tigecycline and gentamicin were both sensitive in $25.5 \%$ of cases of Streptococci infections . In combination, linezolid vancomycin erythromycin in $25 \%$ and linezolid vancomycin cephalexin in $25 \%$.

\section{Staphylococci :}

In combination gentamicin and cefuroxime were sensitive in $25 \%$, and rifampacin and clindamycin in $25 \%$ towards Staphylococci.

\section{P.aeruginosa :}

Meropenem was sensitive in $12.5 \%$ cases P.aeruginosa. In combination doripenem and levofloxacin, ciprofloxacin and nitilmicin, colistin and meropenem, imipenem and ciprofloxacin, colistin and ciprofloxacin, levofloxacin and ciprofloxacin , and ciprofloxacin meropenem, tricycline are in $12.5 \%$ of cases.

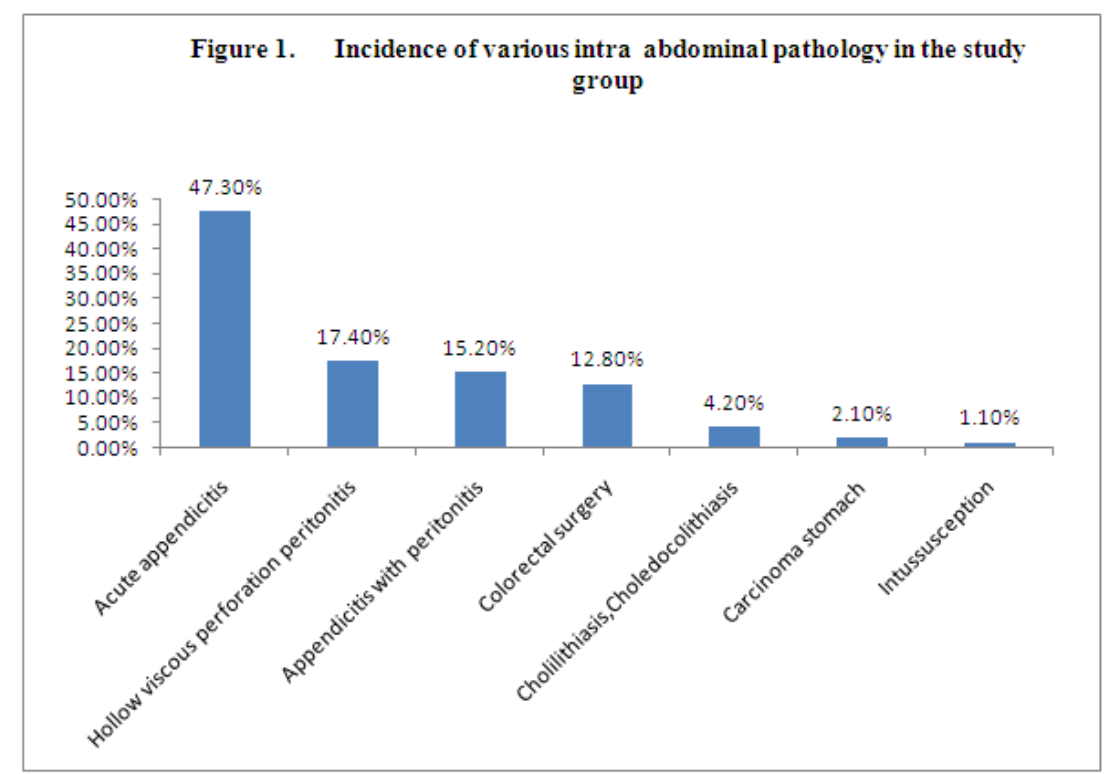

Acute appendicitis were seen in $47.3 \%$. Hollow viscous Perforation peritonitis in $17.4 \%$, appendicitis with peritonitis in $15.2 \%$, Colorectal surgery in $12.8 \%$, Cholilithiasis, Choledocolithiasis in 4.2\%, Carcinoma stomach in $2.1 \%$ and

Intussusception in $1.1 \%$. 
Microbial Spectrum of Intra-Abdominal Pathology of Patients Requiring Surgical Intervention....

Table No.1. Organisms isolated in pathology of study group

\begin{tabular}{|l|l|l|l|l|l|l|l|l|l|}
\hline Organisms & $\begin{array}{l}\text { Acute } \\
\text { appen } \\
\text { dicitis }\end{array}$ & $\begin{array}{l}\text { Hollow } \\
\text { viscous } \\
\text { perforatio } \\
\text { n peritonitis }\end{array}$ & $\begin{array}{l}\text { Appendiciti } \\
\text { s with } \\
\text { peritonitis }\end{array}$ & $\begin{array}{l}\text { Colorecta } \\
\text { ISurgery }\end{array}$ & $\begin{array}{l}\text { Cholilithiasis, } \\
\text { Choledocolit } \\
\text { hiasis }\end{array}$ & $\begin{array}{l}\text { Carcinom } \\
\text { a stomach }\end{array}$ & $\begin{array}{l}\text { Intussce } \\
\text { ption }\end{array}$ & Total & Percentage \\
\hline E.coli & 33 & 8 & 9 & 5 & 1 & 0 & 1 & 57 & 61.3 \\
\hline $\begin{array}{l}\text { K.pneumonia } \\
e\end{array}$ & 6 & 4 & 3 & 4 & 1 & 0 & 0 & 18 & 19.2 \\
\hline Enterococcus & 7 & 2 & 3 & 5 & 0 & 0 & 0 & 17 & 18.3 \\
\hline P.aeruginosa & 3 & 2 & 3 & 0 & 0 & 0 & 0 & 8 & 8.6 \\
\hline $\begin{array}{l}\text { Acinetobacte } \\
r\end{array}$ & 3 & 3 & 0 & 1 & 0 & 1 & 0 & 7 & 7.5 \\
\hline Enterobacter & 0 & 2 & 0 & 0 & 2 & 1 & 0 & 5 & 5.4 \\
\hline Streptococci & 2 & 0 & 0 & 0 & 1 & 1 & 0 & 4 & 4.3 \\
\hline $\begin{array}{l}\text { Staphylococc } \\
i\end{array}$ & 0 & 0 & 1 & 1 & 0 & 0 & 0 & 2 & 2.2 \\
\hline
\end{tabular}

The commonest bacteria isolated was E.coli with $61.3 \%$,followed by K.pneumoniae with $19.2 \%$ and Enterococcus with $18.5 \%$. Enterococcus spp. were isolated with $18.3 \%$ which is followed by P.aeruginosa (8.6\%), Acinetobacter spp. (7.5\%), Enterobacter spp. (5.4\%), Streptococci (4.3\%) and Staphylococci (2.2\%).

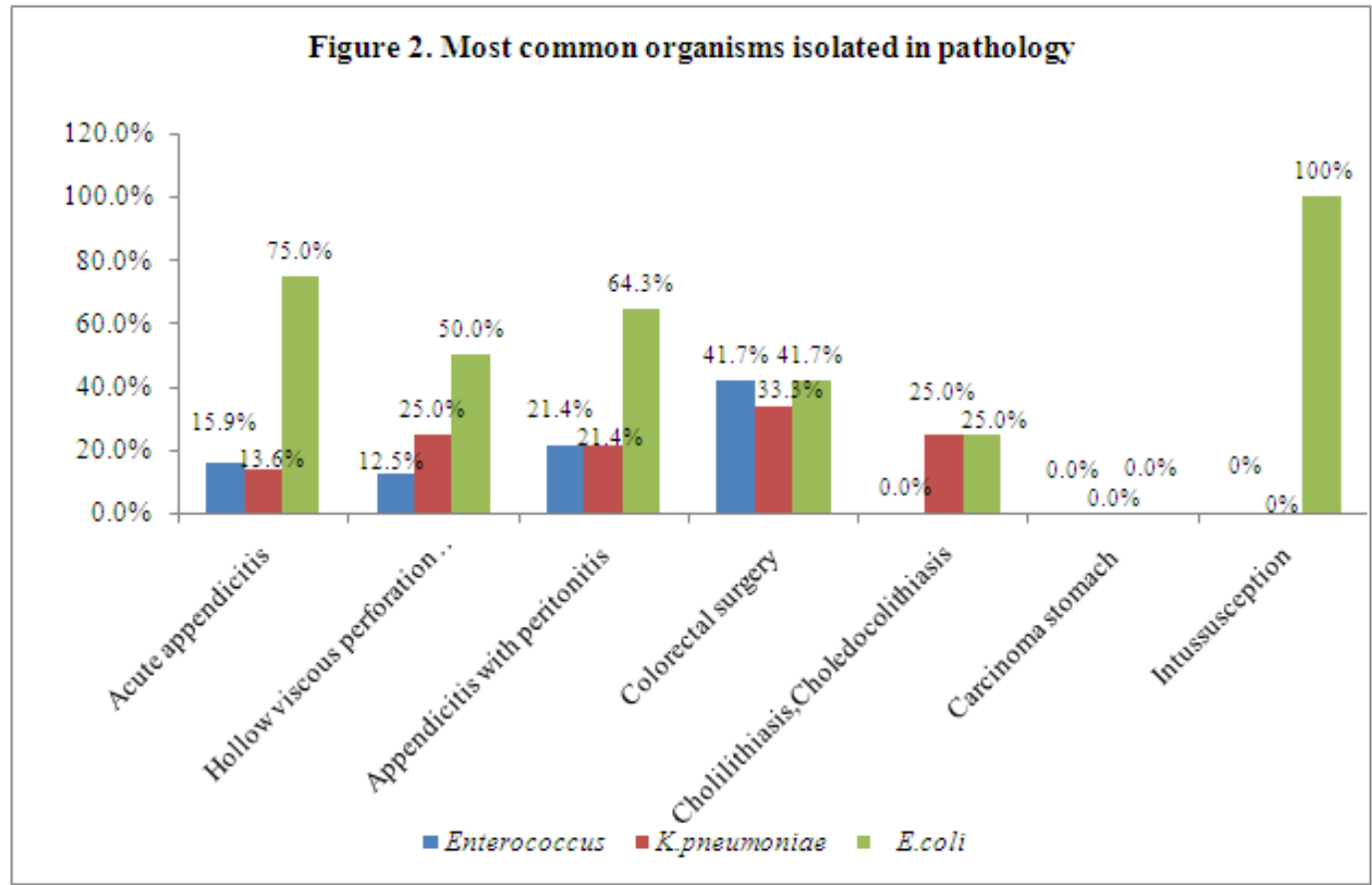

In $47.3 \%$ of cases of acute appendicitis. E.coli dominated with $75 \%$. Followed by Enterococcus spp. with 15.9\%.In $17.4 \%$ of cases of hollow viscous perforation peritonitis, E.coli dominated with 50\%. Followed by K.pneumoniae with $25 \%$. In $15.2 \%$ of cases of acute appendicitis with peritonitis, E.coli dominated with $64.3 \%$. Followed by K.peumoniae and Enterococcus with $21.4 \%$. In $12.8 \%$ of cases of colorectal surgery, E.coli and Enterococcus spp. were seen in $41.7 \%$ of cases. Followed by K. pneumoniae with $33.3 \%$. 


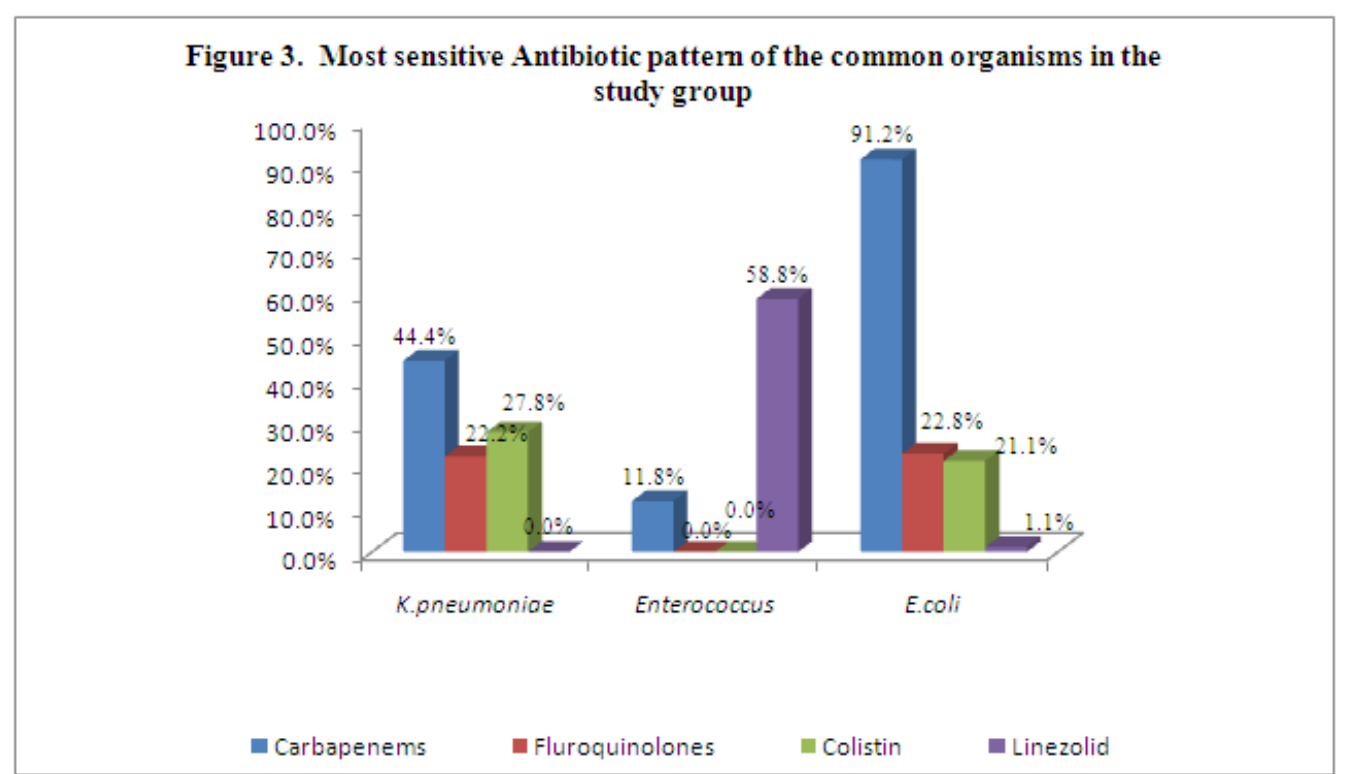

E.coli was sensitive to carbapenems in $91.2 \%$ of cases, $22.8 \%$ to fluroquinolones and $21.1 \%$ to colistin. K.pneumoniae was sensitive to carbapenems in $44.4 \%$ of cases, $27.8 \%$ to colistin and $22.2 \%$ to fluroquinolones. Enterococcus spp. was sensitive to linezolid in $58.8 \%$ of cases and $11.8 \%$ to carbapenems.

\section{Discussion}

The treatment of patients with intra-abdominal infections must be approached with calculated strategy because mortality rates for these patients can approach a high percentage. Intra abdominal infections are typically managed using surgical intervention in conjunction with antibiotic therapy, but selecting appropriate initial empiric therapy is crucial.

The most common source of infection in community acquired intra-abdominal infections is the appendix, followed by the colon, and then the stomach.

Intra abdominal pathology

In this study, the most common pathology was acute appendicitis which accounted for $47.3 \%$ for which the frequent surgery done was laparoscopic appendicectomy. Hollow viscous perforation peritonitis accounted to about $17.4 \%$ followed by appendicitis with peritonitis with $15.2 \%$ and Colorectal surgery with $12.8 \%$. Cholilithiasis, Choledocolithiasis were present in $4.2 \%$ of cases and carcinoma stomach in $2.1 \%$ of cases. Intussusception accounted to about $1.1 \%$.

In a study by K. Krobot et al.(2004) ${ }^{3}$, it was found that appendicular pathology was the most common intra-abdominal pathology with 38\%, colonic pathologies accounted with $26 \%$, duodenal pathologies with $23 \%$ and biliary pathologies for which cholecystectomy was done accounted to about $6 \%$.

In a different study by Sartelli et al. (2012) ${ }^{6}$ it was shown that the most common intra-abdominal infections were appendicitis. It accounted to about $38.4 \%$ of cases for which appendicectomy was done. Cholecystitis was see in $14.4 \%$ of cases for which cholecystectomy surgry was done. Gastroduodenal suturing was done for gastroduodenal perforation which accounted for $8.1 \%$. Other common pathologies accounted in $4.9 \%$ of cases.

These articles are in concordance with the results obtained from the present study.

Organisms isolated from intra abdominal pathology

In the present study, the commonest bacteria isolated was E.coli with $61.3 \%$,followed by K.pneumoniae with $19.2 \%$ and Enterococcus with $18.5 \%$. Enterococcus spp. were isolated with $18.3 \%$ which is followed by P.aeruginosa (8.6\%), Acinetobacter spp.(7.5\%), Enterobacter spp. (5.4\%),Streptococci (4.3\%) and Staphylococci $(2.2 \%)$.

In a study by, D.H Wittmann et al. (1991 $)^{4}$; Out of 900 isolates from intra abdominal pathologies, E.coli was the most common aerobe isloated. It was isloated from about $50 \%$ patients with intra abdominal pathologies. K. pneumoniae was isloated from about $12.2 \%$ patients.Both Enterobacter spp. and Pseudomonas spp. accounted in less than $11.1 \%$ cases of intra abdominal pathology.In anaerobic organisms Bacteroides was isolated in about more than $66.6 \%$ cases.

In a similar study by K. Krobot et al. (2004) $)^{3}$, E.coli was the most common organism isolated which accounted to about $47 \%$ of cases and was followed by K.pneumoniae with $7 \%$. Both Enterobacter spp. and P.aeruginosa accounted for about $4 \%$ of cases 
In another study by Sartelli et al. $(2012)^{6}$; aerobic gram negative bacteria accounted to about $70.6 \%$, which mainly was E.coli in about $45 \%$ of cases. E.coli resistant to third generation cephalosporins accounted to about $5 \%$. K.pneumoniae were isolated in $7.9 \%$ of cases. K.pneumoniae resistant to third generation cephalosporins accounted to $2.7 \%$ of cases. Enterobacter spp. with $4 \%$, Pseudomonas with $4.6 \%$ Proteus with $2 \%$ and others with $7 \%$ were also isolated. In case of aerobic gram positive bacteria Staphylococci accounted to about $3.1 \%$ and Streptococci with $6.9 \%$.

In another study by Neetu Shree et al. (2014) $)^{7}$, Escherichia coli $(43.5 \%)$ emerged as the most predominant pathogen followed by Klebsiella spp. (25.4\%), while Bacteroides fragilis emerged as the predominant anaerobe. Acinetobacter spp.(7.4\%), P. aeruginosa (5.5\%), Enterobacter $\mathrm{spp}(1.8 \%)$, Citrobacter spp. $(0.9 \%)$ and Proteus vulgaris $(0.9 \%)$ were also isolated.

These studies, which were done during different decades, still states that E.coli is the most common organism to be isolated from intra abdominal pathology.

In this study, in $47.3 \%$ of cases of acute appendicitis, E.coli dominated with $75 \%$. Followed by Enterococcus spp. with $15.9 \%$.In $17.4 \%$ of cases of hollow viscous perforation peritonitis, E.coli dominated with $50 \%$. Followed by K.pneumoniae with $25 \%$.In $15.2 \%$ of cases of acute appendicitis with peritonitis, E.coli dominated with $64.3 \%$. Followed by K.peumoniae and Enterococcus spp. with $21.4 \%$.In $12.8 \%$ of cases of colorectal surgery, E.coli and Enterococcus were seen in $41.7 \%$ of cases. Followed by K. pneumoniae with 33.3\%.In a study by M. Guembe et $\mathrm{al}^{5}$. (2003-2007), total of 572 aerobic and facultative gram-negative bacilli were isolated from intra-abdominal infections in 510 patients. E.coli (52\%) was the most common isolated species, followed by Klebsiella spp. (16\%), Proteus mirabilis (6.3\%) and Enterobacter spp. (6.3\%). Among the non-fermenters, $P$. aeruginosa was the most common isolated species $(6.8 \%)$.

In a different study by Neetu Shree et al. $(2014)^{7}$, a total of 108 strains of aerobic bacteria and 22 strains of anaerobic bacteria were isolated during the present study. Three Candida spp. were also isolated. The 92 aerobic Gram negative bacilli (GNB) isolates comprised of 47 (43.5\%) E. coli, Klebsiella spp. $27(25.4 \%)$, Acinetobacter spp. 08 (7.4\%), P.aeruginosa 06 (5.5\%), Enterobacter spp. 02 (1.8\%), Citrobacter spp. 01 (0.9\%) and Proteus vulgaris $01(0.9 \%)$.

These studies also show that acute appendicitis, the most common intra abdominal pathology, E.coli was the most common organism isolated from intra abdominal pathology.

Most sensitive antibiotic pattern

In the present study, E.coli was sensitive to carbapenems in $91.2 \%$ of cases, $22.8 \%$ to fluroquinolones and $21.1 \%$ to colistin. K. pneumoniae was sensitive to carbapenems in $44.4 \%$ of cases, $27.8 \%$ to colistin and $22.2 \%$ to fluroquinolones. Enterococcus spp. was sensitive to linezolid in $58.8 \%$ of cases and $11.8 \%$ to carbapenems.

In a study by D.H Wittmann et al. $(1991)^{4}$, Cefotaxime, cefoperazone and moxalactam were sensitive in $100 \%$ of cases for E.coli. this was followed by cefotetan with $99 \%$ and imipenem with $98 \%$.Ceftazidime was sensitive in $100 \%$ of cases which were followed by cefotaxime and cefotetan with $99 \%$ sensitivity for K.pneumoniae.

In a different study by Santosh Saini et al. (2004) $)^{8}$, it was found that, from the study ceftizoxime was sensitive to E.coli in about $77.7 \%$ of cases followed by cefotaxime with $66.6 \%$ and ciprofloxacin with $51.8 \%$.Both ceftizoxime and cefotaxime were sensitive to K.pneumoniae in about $83.3 \%$ of cases followed by amikacin with $55.5 \%$ of sensitivity. Ceftizoxime, amikacin and ciprofloxacin were sensitive in $35.2 \%$ of cases for P.aeruginosa followed by cefotaxime with $23.5 \%$ sensitivity.

In three different studies by Villages et al. $(2011)^{9}$, Hauser et al.(2007), Chaudhuriet al.(2009) ${ }^{10}$, Imipenem, tigecycline, and colistin appeared as the most active drugs against E. coli and Klebsiella spp. (100\% susceptible).

These different studies show that, the sensitivity pattern of the most common organisms like E.coli, K. pneumonia and Enterococcus have changed from cephalosporins to carbapenems. Our study also shows that carbapenems are the most sensitive drug.

From the present study group, E.coli was most sensitive to carbapenems with $91.2 \%$ and $22.8 \%$ to cephalosporins.K.pneumoniae have maximum sensitivity to carbapenems and $27.8 \%$ to colistin and $22.2 \%$ to fluroquinolones.Enterococcus spp. had maximum sensitivity to linezolid with $58.8 \%$.

Taking into account of all these, it appears that carbapenems, linezolid, colistin and fluroquinolones should be considered as the $1^{\text {st }}$ line calculated empirical therapy in treating surgical cases of intra abdominal pathology. 


\section{Conclusion}

The study emphasizes need for antimicrobial susceptibility testing of clinically significant isolates not only as a routine procedure but also on periodic basis, specially when no definitive resistance or susceptibility patterns are available in a given geographic location.

A calculated empiric therapy would reduce the chances of microorganisms in developing resistance to anti microbial therapy.

\section{Acknowledgement}

First and foremost, praises and thanks to the God, the Almighty, for His showers of blessings throughout my research work to complete the research successfully.

This thesis appears in its current form due to the assistance and guidance of several people. I would therefore like to offer my sincere thanks to all of them.

I would like to extend my sincere gratitude to Indian Council of Medical Research for providing me a great opportunity of carrying out a research under the short Term Studentship Program.

I am also extremely indebted to my guide Dr.Vergis Paul, Professor of General Surgery for providing necessary information, constructive criticism and his extensive discussions and resources to accomplish my research work. I thank Dr.Jomine Jose, Associate Professor of General Surgery for his valuable advice around my work. I would also like to extend my regards to Department of General Surgery and the Medical Records Department of the hospital for permitting me with the medical records information. I would like to acknowledge my alma mater for the encouragement and support I received to do the study.

I also put it on note my sincere gratitude to Dr. Anna Mathew, Professor of Pharmacology and Research Coordinator for helping me out with the study.

Last but not the least, I would like to pay high regards to my family for their sincere encouragement and support.

\section{References}

[1]. John. E. Mazuski.,SurgClin N Am,Intra-abdominal infections, doi:10.1016/j.suc.2008.12.001,(2009) $421-437$.

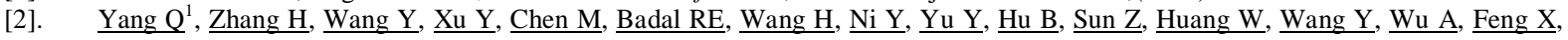

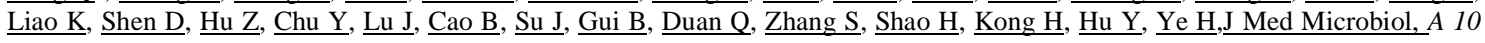
year surveillance for antimicrobial susceptibility of Escherichia coli and Klebsiella pneumoniae in community- and hospitalassociated intra-abdominal infections in China,doi: 10.1099/jmm.0.059816-0. Epub 2013 June 5,'2013 Sep;62(Pt 9):1343-9.

[3]. K. Krobot. D. Yin . Q. Zhang. S. Sen A. Altendorf-Hofmann . J. Scheele . W. Sendt,Eur J ClinMicrobiol Infect Dis, Effect of inappropriate initial empiric antibiotic therapy on outcome of patients with community-acquired intra-abdominal infections requiring surgery,(2004) 23: 682-687.

[4]. D.H Wittmann, J.M Bergstein, C.Frantzides, , Infection 19 (1991) Suppl. 6 @ MMV MedizinVerlag GmbH Mfinchen, Mfinchen 1991,Calculated empiric antimicrobial therapy for mixed surgical infections, 1991

[5]. M. Guembe E. Cercenado L. Alcalá M. Marín R. Insa and E. Bouza,Evolution of antimicrobial susceptibility patterns of aerobic and facultative gram-negative bacilli causing intra-abdominal infections: results from the SMART studies 2003-2007. Servicio de Microbiología Hospital General Universitario Gregorio Marañón Madrid (Spain), Rev EspQuimioter 2008;21(3):166-173.

[6]. Sartelli et al. World Journal of Emergency Surgery 2012, 7:15. Complicated intra-abdominal infections in Europe: preliminary data from the first three months ofthe CIAO Study,http://www.wjes.org/content/7/1/15.

[7]. Neetu Shree, Balvinder S. Arora, Ravinder S. Mohil1, Dimple Kasana, InduBiswal. Departments of Microbiology, 1Surgery, VardhmanMahavir Medical College \&Safdarjang Hospital, New Delhi, India. Agarwal N, Saha S, Srivastava A, Chumber S, Dhar A, Garg S, Bacterial profile and patterns of antimicrobial drug resistance in intra-abdominal infections: Current experience in a teaching hospital, Peritonitis: 10 years' experience in a single surgical unit. Trop Gastroenterol 2007;28:117-20.

[8]. Santosh Saini ; Naveen Gupta; Aparna ${ }^{\mathrm{I}}$; Lokveer'; M.S. Griwan ${ }^{\mathrm{II}}$.. Department of Microbiology, Pt. B.D. Sharma PGIMS, Rohtak, India IIDepartment of Surgery, Pt. B.D. Sharma PGIMS, Rohtak, India,Surgical infections: a microbiological study,Brazilian Journal of Infectious Diseases, Braz J Infect dis vol.8 no.2 Salvador Apr. 2004, http://dx.doi.org/10.1590/S141386702004000200001 .

[9]. Villegas MV, Blanco MG, Sifuentes-Osornio J, Rossi F. Increasing prevalence of extended-spectrum-beta lactamase among gramnegativebacilli in latinamerica - 2008 update from the study for monitoring antimicrobial resistance trends (smart), BrazJInfectDis2011;15:34-9.

Hauser SP, Bouchillon SK, Hoban DJ, Badal RE, Hsueh PR, Paterson DL,Emergence of high levels of ESBL producing gram negative bacilli in the asia pacific region: Data from the study for monitoring antimicrobial resistance trends( SMART) programme, 2007,Antimicrob Agents Chemother 2009;53:3280-4.

[10]. Chaudhuri BN, Rodrigues C, Balaji V, Iyer R, Sekar U, Wattal C, et al. Incidence of ESBL producers amongst Gram-negative bacilli isolated from intra-abdominal infections across India (based on SMART study, 2007 data), J Assoc Physicians India 2011;59:287-92. 